\title{
Feasibility of treatment free remission with generic imatinib: Results of GIFT-in-CML-CP study
}

\author{
Deepak Goni ${ }^{1}$, Arihant Jain ${ }^{1}$, Nishant Jindal ${ }^{1}$, Ram Nampoothiri ${ }^{1}$, Deepesh Lad ${ }^{1}$, Shano \\ Naseem $^{1}$, Gaurav Prakash ${ }^{1}$, Alka Khadwal ${ }^{1}$, Neelam Varma ${ }^{1}$, and Pankaj Malhotra ${ }^{1}$ \\ ${ }^{1}$ PGIMER
}

October 1, 2020

\begin{abstract}
Both innovator and generic imatinib are approved for the treatment of Chronic Myeloid Leukemia-Chronic phase (CML-CP). Currently, there are no studies on the feasibility of treatment free remission (TFR) with generic imatinib. In a single center prospective Generic Imatinib Free Trial - in -CML-CP (GIFT-in-CML-CP) study, twenty-six patients on generic imatinib for more than 3 years and in sustained deep molecular response (BCR ABLIS $<0.01 \%$ for more than 2 years) were included. After treatment discontinuation, patients were monitored with complete blood count and BCR ABLIS by real time quantitative PCR monthly for one year and three monthly thereafter. Generic imatinib was restarted at single documented loss of major molecular response. At a median follow-up of 20 months (range, 4-34 months), $42.3 \%$ patients ( $\mathrm{n}=11$ ) continued to be in TFR. Estimated TFR at 1 year was $44 \%$. All patients restarted on generic imatinib regained major molecular response. On multivariate analysis, attainment of complete molecular remission (CMR) prior to TFR trial was predictive of $\mathrm{TFR}[\mathrm{p}=0.022$, HR $0.284(0.096-0.837)]$. We conclude that, generic imatinib can be safely discontinued in CML-CP patients who are in deep molecular remission.
\end{abstract}

Fundi Feasibility of treatment free remission with generic imatinib: Results of GIFT-in-CMLCP study

Deepak Goni ${ }^{1}$,

Arihant Jain ${ }^{1}$,

Ram Nampoothiri, ${ }^{1}$

Nishant Jindal ${ }^{1}$,

Deepesh $\operatorname{Lad}^{1}$,

Shano Naseem ${ }^{2}$,

Gaurav Prakash ${ }^{1}$

Alka Khadwal, ${ }^{1}$

Neelam Varma, ${ }^{2}$

Pankaj Malhotra ${ }^{1^{*}}$

${ }^{1}$ Department of Internal Medicine, ${ }^{2}$ Department of Haematology, Post Graduate Institute of Medical Education and Research, Chandigarh, India

*Correspondence 
Prof. Pankaj Malhotra

Dept of Internal Medicine

PGIMER Chandigarh 160012

India

Email: malhotrapankaj@hotmail.com

Phone number: +911722756671

Funding : This study was funded by the institute.

Running Head : Treatment free Remission with generic Imatinib

Key Words : Generic , Imatinib, Treatment Free Remission

Word Count :1327, Figures : 1, Tables :2

What is already known about the subject?

Treatment free remission is feasible with tyrosine kinase inhibitors in patients of Chronic Phase Chronic Myeloid Leukemia.

What this study adds?

The use of generic imatinib is widely prevalent throughout the globe, although its efficacy and tolerability is still debated.To the best of our knowledge this is the first trial on treatment free remission in patients on generic imatinib.

Conflict of Interest : All authors declare that there is no conflict of interest.

Ethics approval : All procedures performed in studies involving human participants were in accordance with the ethical standards of the institutional and/or national research committee and with the 1964 Helsinki declaration and its later amendments or comparable ethical standards. Ethics approval was sought from the institute ethics committee

Informed consent : Informed consent was obtained from all individual participants included in the study.

Principal Investigator Statement : The authors confirm that the Principal Investigator for this paper is Deepak Goni and he had direct clinical responsibility for patients.

Author Contributions : DG and RN conducted the study,NJ did the statistical analysis,PM,DL,AK,GP and AJ planned the study and drafted the final manuscript,NV and SN provided the laboratory support.

Hosted file

BJCP_Manuscript with figures and tables.pdf available at https://authorea.com/users/362983/

articles/483917-feasibility-of-treatment-free-remission-with-generic-imatinib-results-

of-gift-in-cml-cp-study 